\title{
Image Transmission over Channels with Bit Errors and Packet Erasures
}

\author{
Pamela C. Cosman, Jon K. Rogers, P. Greg Sherwood, and Kenneth Zeger *
}

\begin{abstract}
We consider a hybrid mixture of an image coder for bit-error channels and an image coder for packet erasure channels. The first scheme uses rate-compatible punctured convolutional $(R C P C) / c y c l i c$ redundancy code $(C R C)$ concatenated channel coding with embedded zerotree wavelet source coding, while the second technique groups trees of wavelet coefficients into fixed-length packets that provide robustness against packet erasures. We demonstrate that the hybrid image coder outperforms either of the two building block methods on certain channels that suffer both packet losses and statistically varying bit errors.
\end{abstract}

\section{Introduction}

The transmission of images across noisy channels is fundamentally important in many applications and is still an active research problem. One basic approach has been to start with a high-performance source coder, and protect its output from errors by adding redundancy (e.g., [1, 5, 6, 12, 13]). Another method has been to design resilience into the source coder so the effect of channel errors is reduced and less channel coding is necessary [2, 3, 7, 8, 9].

Many data transmission environments are characterized by unknown and highly varying channel conditions. In mobile wireless environments, it can be difficult to accurately measure channel conditions and adapt the coding. Likewise, in a broadcast channel each receiver experiences a different channel, so designing a good coding scheme for all receivers may mean designing for the worst case. Other systems experience transmission errors in the form of packet loss due to buffer overflow, mis-routing, or unacceptably long arrival delays. These situations are typically modeled by the packet erasure channel. A mobile receiver may experience both packet losses and also bit errors on packets

* The authors are with the Department of Electrical and Computer Engineering, University of California at San Diego, La Jolla, CA 92093-0407. email: \{cosman, jkrogers, sherwood, zeger\}@code.ucsd.edu. This work was supported in part by the National Science Foundation. which are not lost. In this paper, we introduce a robust hybrid encoding scheme to address this combination of channel impairments.

We present a hybrid coder which combines the block error control method of $[12,13]$ with the zerotree packetization method of $[8,9]$. The hybrid scheme provides more robust performance over varying channel conditions. Specifically, we measure the performance improvement of the hybrid coder on Jakes' [4] model for a fading channel combined with a packet erasure model. This model is used to simulate source data being sent to a transmitter (wireline link) and then broadcast to a mobile receiver (wireless link). The particular hybrid coding model presented is not claimed to be optimal, but rather was chosen as an example of the potential improvement possible using this new design approach.

\section{The Hybrid Coder}

The excellent compression performance of the Set Partitioning In Hierarchical Trees (SPIHT) [10] source coder comes at the expense of a significant sensitivity to errors. Errors often lead to a complete loss of synchronization in the decoder due to the use of variable length coding. In $[12,13]$, SPIHT is followed by a strong concatenated channel code (RCPC/CRC) which lowers the probability of decoding errors, thereby providing protection against synchronization loss. Also, the CRC allows detection of uncorrected packets so the source decoder can stop decoding before errors propagate and corrupt the image. For a binary symmetric channel (BSC) of known error rate, this method often produces acceptable image quality due to the progressive nature of the source coder.

Source coding can be designed to provide noise robustness without explicit error-correction coding. The Packetized Zerotree Wavelet (PZW) coder [8] provides robustness by producing a compressed image datastream consisting of independently decodable packets. PZW is an error-resilient variation on the Embedded Zerotree Wavelet (EZW) and SPIHT coders [10, 11]. Groups of wavelet coefficient trees are placed together into fixed length packets 
(typically hundreds of bits) with a 16-bit CRC for error detection. At the receiver, packets received with detected errors are discarded; others are decodable independent of any other packet. Missing trees are concealed by interpolating missing low-low band wavelet coefficients; missing higher band coefficients are set to zero prior to inverse wavelet transforming the array.

The growing and pruning of coefficient trees in order to fit fixed-length packets, as well as the addition of a small header, induce some performance loss, but they provide robustness against packet loss. Errors cannot propagate beyond packet boundaries. Synchronization is not lost if packets are dropped. Packets are of equal importance; given a certain packet loss rate; it matters little to the final PSNR which packets were lost.

Both PZW and SPIHT+RCPC/CRC coders have difficulties on channels with varying conditions. PZW will suffer many packet losses as the bit error pattern becomes more uniform. A single error in a packet will lead to a packet erasure. The SPIHT+RCPC/CRC coder must use a source/channel code rate allocation designed for the worst case channel because channel codes typically transition rapidly from the designed performance to uncoded performance (and even worse) as the channel degrades. This sacrifices performance when the channel is clear - much of the available rate is spent on channel coding (especially for highly variable channels). Since the SPIHT source decoder requires an uninterrupted stream of source bits to maintain synchronization, the SPIHT+RCPC/CRC coder is also sensitive to packet erasures which occur early in the transmission.

Our proposed hybrid coder uses the PZW algorithm for source coding. Each packet is protected by the RCPC/CRC code from $[12,13]$. The RCPC/CRC code is designed for channel conditions in the middle of the expected range. Some bit interleaving is also used to improve performance of the RCPC codes on the bursty channels. The resulting datastream is better suited to handle a larger range of channel conditions. Where a packet erasure would truncate the bitstream using the SPIHT+RCPC/CRC coder, the hybrid system can use all received packets. PZW on its own cannot handle arriving packets with errors, but the hybrid has the RCPC/CRC code to correct bit errors making received packets useful to the source decoder. In essence, the two approaches used together are intended to help fix the weaknesses of each other.

\section{The Channel Model}

We model the channel as a discrete channel with memory, combined with a packet erasure channel. The discrete component uses Jakes' [4] model to simulate binary phase-shift keying (BPSK) transmission over a flat-fading
Rayleigh channel. This model is characterized by two parameters - the average received SNR $(\overline{\mathrm{SNR}})$, which determines the average bit error rate, and the normalized Doppler spread $f_{D}=f_{m} / R$ (i.e., the maximum Doppler shift normalized by the data rate), which determines how quickly the channel changes over time (large values of $f_{D}$ indicate fast changes over time, leading to short bursts). The channel model was selected to accurately simulate the fading channels common in mobile wireless environments. The packet erasure portion of the channel was modeled using the probability of packet erasure, $p_{\text {erasure }}$, and a packet burst length parameter $L$, which is the number of consecutive erased packets. Within the model, the source output is divided into groups of $L$ packets, and each group is erased with probability $p=\left(p_{\text {erasure }}\right) / L$ so that the overall erasure rate is $p_{\text {erasure }}$ regardless of the burst length. This model was chosen to simulate a wireline network in which packet losses occur due to a combination of queue overflow, mis-routing, and delay constraints (for video). The packet burst length parameter allows the model to produce bursty packet losses typical of network impairments. To test the effect of correlated packet erasures, bursts of lengths $L=1$ (i.e., independent packet erasures) and $L=10$ were simulated for each erasure rate.

\section{Results and Conclusions}

To compare the three algorithms for robustness, we chose a range of channel conditions with various discrete error and packet error parameters. The Rayleigh fading channel ranged from low-power slow-fading $\left(f_{D}=10^{-5}\right.$, $\overline{\mathrm{SNR}}=10 \mathrm{~dB})$ to moderate-power fast-fading $\left(f_{D}=2 \times\right.$ $\left.10^{-4}, \overline{\mathrm{SNR}}=20 \mathrm{~dB}\right)$. Packet erasure parameters tested were combinations of erasure rate $p_{\text {erasure }}=\{0.01,0.1\}$ and burst lengths $L=\{1,10\}$. The SPIHT+RCPC/CRC coder and the hybrid coder were each optimized for a fixed channel near the middle of the range $(\overline{\mathrm{SNR}}=13 \mathrm{~dB}$, $f_{D}=10^{-4}$, no packet erasure). The optimization criterion was the minimization of the mean decoded MSE. No optimization was done for the PZW coder; one could alter various parameters such as the packet length, wavelet decomposition depth, and tree branching rate to optimize for a particular channel, but it is not straightforward to do this. The algorithms were then tested using the $512 \times 512$ Lena image with total transmission rate fixed at $0.25 \mathrm{bpp}$. Any bit interleaving (for SPIHT+RCPC/CRC and hybrid coders) was limited to a maximum depth of 70 bits. Each channel condition was tested with a minimum of 1000 independent trials and as many as 3000 trials on the slowest channels.

Initial tests used channels with only the Rayleigh fading component to analyze robustness to discrete errors. SPIHT+RCPC/CRC generally showed improved performance (lower mean MSE) for higher received $\overline{\mathrm{SNR}} \mathrm{s}$ as well 


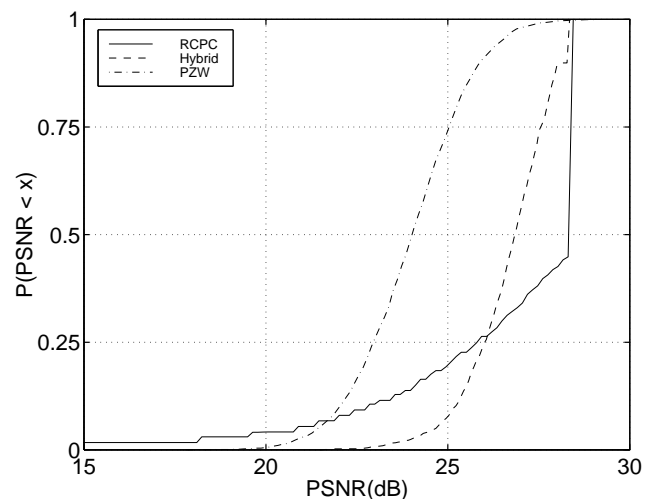

(a) Packet Erasure Rate 0.01, Burst Length 1

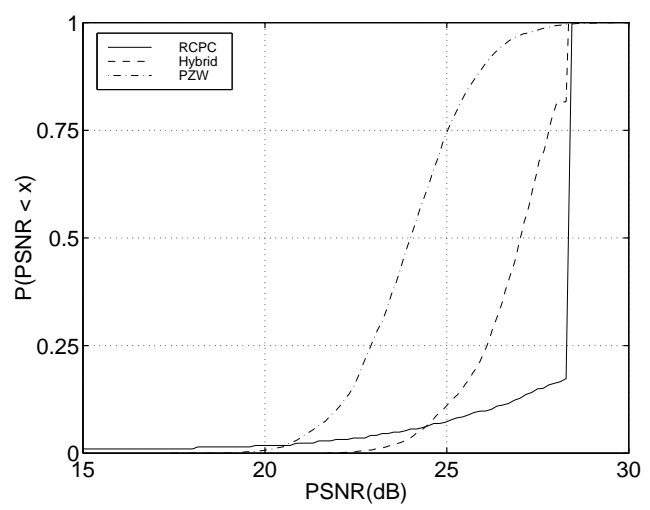

(c) Packet Erasure Rate 0.01, Burst Length 10

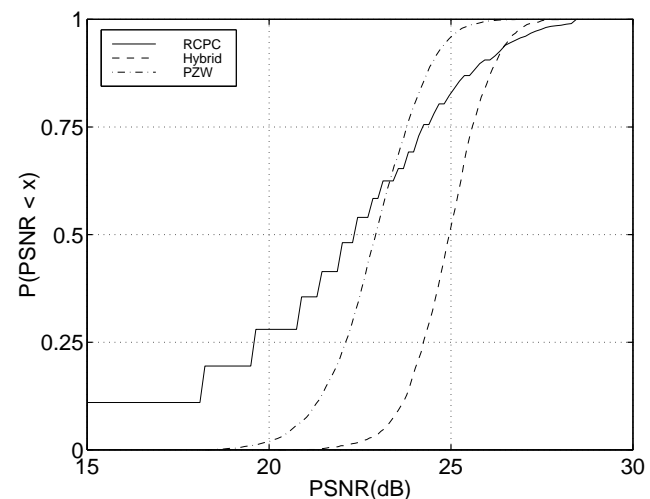

(b) Packet Erasure Rate 0.10, Burst Length 1

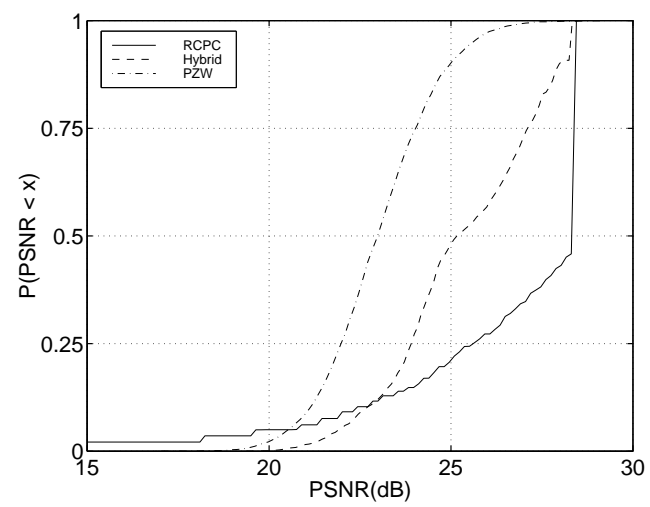

(d) Packet Erasure Rate 0.10, Burst Length 10

\section{Figure 1. Cumulative distributions of decoded PSNR for the $512 \times 512$ Lena image over channels with varying packet erasure parameters and fading parameters $f_{D}=10^{-4}, \overline{\mathrm{SNR}}=12 \mathrm{~dB}$. (Total transmission rate 0.25 bpp.)}

as for faster channels (larger Doppler spread). On faster channels, the interleaver is effective in making the error more uniform over the data. This kind of error pattern can often be corrected by the RCPC code. In contrast, the performance of the PZW coder degrades for faster channels because the errors are less bursty - more packets are lost for a given average error rate. The hybrid coder, which can tolerate a few lost packets but has the ability to correct some bit errors, showed competitive if not superior performance for all tested channels (based on the mean MSE).

In a second set of tests, we fixed the Rayleigh channel parameters $\left(f_{D}=10^{-4}\right.$ and $\left.\overline{\mathrm{SNR}}=12 \mathrm{~dB}\right)$ and varied the packet erasure settings. Because the SPIHT+RCPC/CRC algorithm must stop decoding at the first uncorrected error or erasure, channels with high erasure rates lead to higher probability of incomplete decoding and lower performance. Also, for a given packet erasure rate, the performance was better on channels with long erasure burst lengths simply because the more uniform erasure patterns have a higher likelihood of the first erasure occurring early in the transmission. For PZW the performance essentially depends only on the number of lost packets and not the particular pattern of losses due to packet erasures or bit erasures, so the performance on channels with packet erasures was similar to that on Rayleigh channels with lower SNR. Again, the hybrid coder's robustness allows it to perform competitively over the range of different packet erasure channels and better in the case where packet erasures are high $\left(p_{\text {erasure }}=0.1\right)$ and packet burst length is low $(L=1)$. 


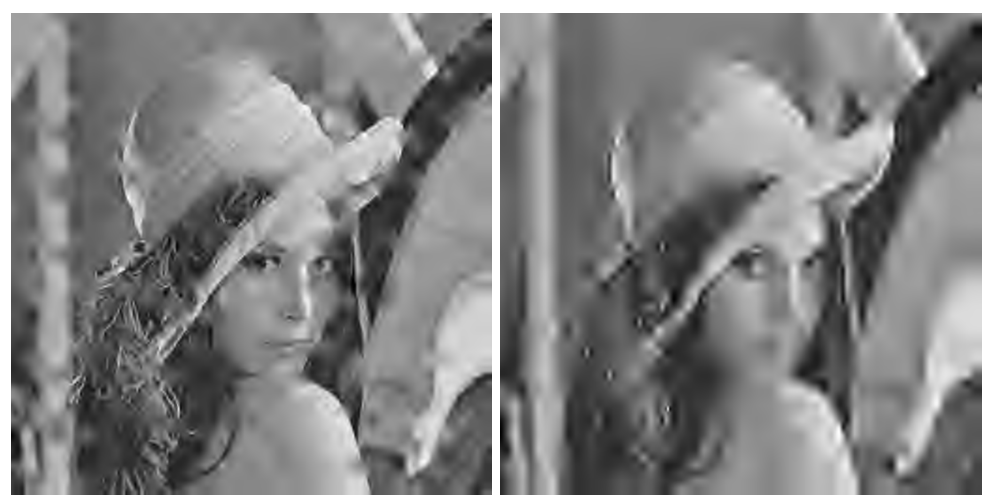

Figure 2. Images displayed here show the visual effects of loss for the hybrid coder (left) vs. the SPIHT+RCPC/CRC coder (right) at equal distortion $(P S N R=23.5 d B)$.

Figures 1(a) - 1(d) are plots of the cumulative distributions of the decoded PSNR for the second group of tests (fixed Rayleigh channel and varying packet erasure parameters). These plots show the overall performance characteristics of each algorithm over the different channels. Curves showing good performance lie near the bottom and right axes, indicating a low probability of decoding at a low PSNR and high probability of decoding at a high PSNR. If the curve for one algorithm lies entirely below and to the right of the curve for another algorithm, then we could conclude that the first algorithm is better. We also need to take into account the fact that the distortion from the channel errors does not manifest itself in the same way for different algorithms. In cases where the total distortion is high, it would be preferable to have that distortion concentrated in a small region of the reconstructed image. The visual effects of the distortion can be somewhat mitigated by using the correlation of neighboring low distortion regions to hide the errors. Alternatively, when the total distortion is low, visual quality will be better if that distortion is spread equally over the image.

Because of the progressive nature of the SPIHT+RCPC/CRC coder, the distortion is evenly distributed. In the high error case, the reconstruction appears blurry because the distortion corresponds to little received high frequency content. Data organization in the hybrid coder is such that the distortion is not distributed equally over the image. Each packet contains information about a number of small spatial regions in the image. If a packet is lost, the distortion will be spatially limited to the areas described by that packet. The quality of data from packets which are successfully decoded will be as good as the initial source coding rate allows. Interpolation of lost coefficients in the low frequency band of the wavelet decomposition is used to further improve the final visual quality. Figure 2 shows two images with equal PSNRs, one from the hybrid coder, and one from the SPIHT+RCPC/CRC coder. One can see, in this high distortion case, that the regions of the image which were successfully decoded by the hybrid method have low distortion, while the SPIHT+RCPC/CRC coder has distortion distributed over the entire image.

This analysis regarding the visual qualities seen for different PSNRs is important when reading the cumulative distribution plots. Taking into account the fact that the hybrid coder is visually better at low PSNR values, we find that the hybrid coder, which may not perform best for any single channel, shows robust performance over a large range of possible channels. In Figure 3, we show sample images representing the median quality expected for the three algorithms over two different channels. The top row of images were sent over a channel with high packet erasure rate and moderate bit errors. The bottom row channel had no packet erasures and long bursts of bit errors. Over these two channels, the hybrid coder is visually more robust than both the SPIHT+RCPC/CRC and PZW. By trading off some performance optimization (the hybrid may not be the best for any particular channel) we have added the flexibility of the coder to perform well over a wide range of channel conditions.

Because severe channels can lead to large variations in decoded image quality over different trials, it is difficult to decisively conclude which coding method is superior. The best choice for any single channel or small range of channel conditions depends on the end application. To design a robust coder, we cannot base the evaluation on a single number such as mean decoded MSE due to the very different shapes of the decoded MSE distributions and the different visual qualities at low PSNR values. However, using the cumulative distribution plots, and the visual results obtained by this research, it is clear that the hybrid performs competitively across all channel conditions and degrades more gracefully under the most severe conditions. 

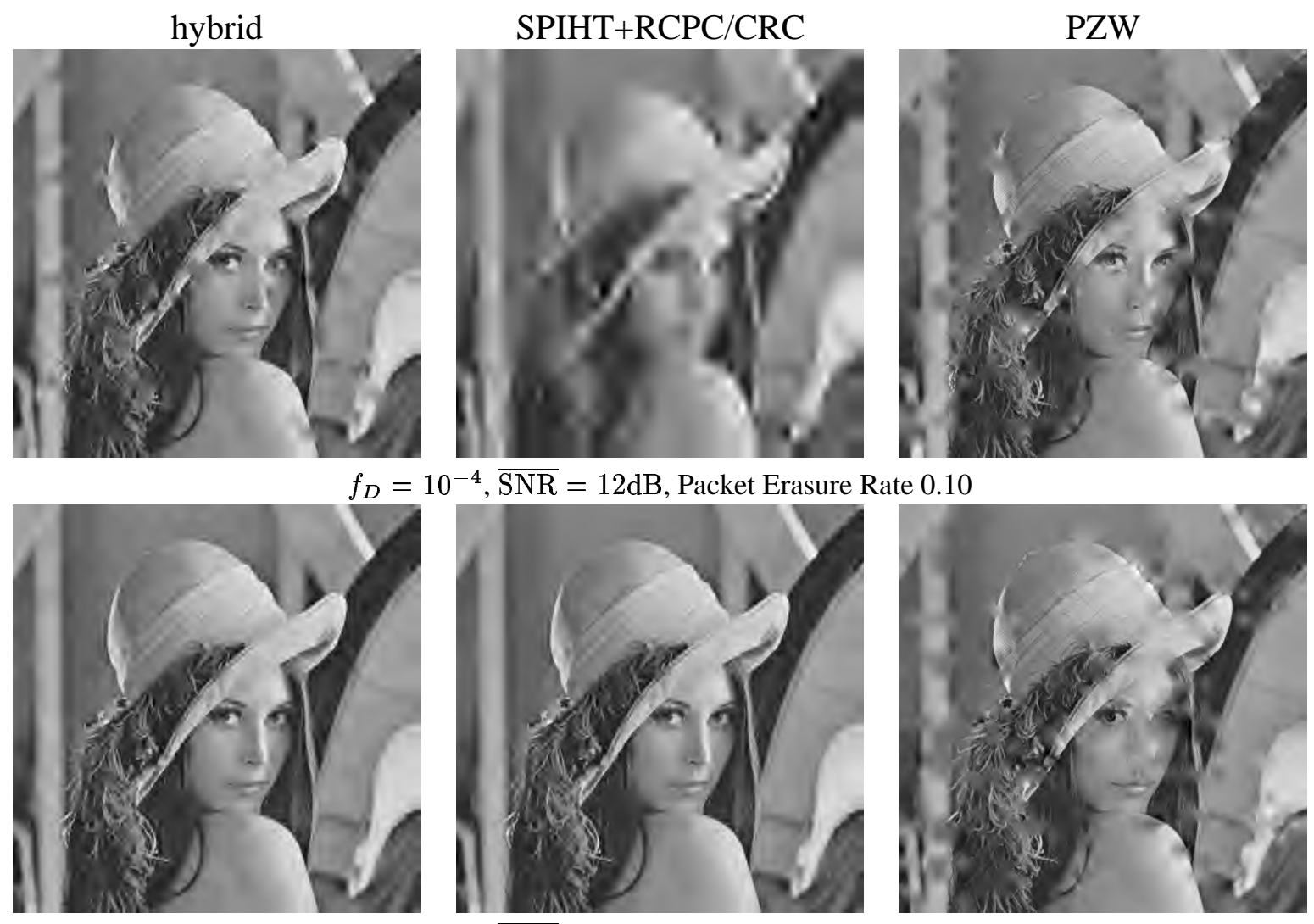

$$
f_{D}=10^{-5}, \overline{\mathrm{SNR}}=10 \mathrm{~dB} \text {, Packet Erasure Rate } 0.00
$$

Figure 3. These Images show the median performance of the three algorithms for two channels. Images in the top row were sent across a channel dominated by packet erasures. The channel for the bottom row of images was a slow-fading low-power channel (high probability of bit errors in long bursts) with no packet erasure. Overall transmission rate was $0.25 \mathrm{bpp}$.

For more detailed numerical results, please visit our web site at http://code.ucsd.edu

\section{References}

[1] V. Chande, H. Jafarkhani, , and N. Farvardin. Joint sourcechannel coding of images for channels with feedback. In Proc. of the IT Workshop, pages 50-51, Feb. 1998.

[2] C. Creusere. A new method of robust image compression based on the embedded zerotree wavelet algorithm. IEEE Trans. on Image Proc., 6(10):1436-1442, Oct. 1997.

[3] V. Crump and T. Fischer. Intraframe low bitrate video coding robust to packet erasure. In J. Storer and M. Cohn, editors, Proc. DCC '97, page 432, 1997.

[4] W. C. Jakes. Microwave Mobile Communications. WileyInterscience, 1974.

[5] B.-J. Kim, Z. Xiong, and W. A. Pearlman. Progressive video coding for noisy channels. In Proceedings ICIP 98, 1998.

[6] I. Kozintsev, J. Chou, and K. Ramchandran. Image transmission using arithmetic coding based continuous error detection. In Proc. DCC '98, pages 339-348, 1998.
[7] H. Man, F. Kossentini, and M. J. Smith. A class of EZW image coders for noisy channels. In Proceedings ICIP 97, volume 3, pages 90-93, 1997.

[8] J. Rogers and P. Cosman. Robust wavelet zerotree image compression with fixed-length packetization. In Proc. DCC '98, pages 412-428, 1998.

[9] J. Rogers and P. Cosman. Wavelet zerotree image compression with packetization. IEEE Sig. Proc. Letters, 5(5):105107, May 1998.

[10] A. Said and W. A. Pearlman. A new, fast, and efficient image codec based on set partitioning in hierarchical trees. IEEE Trans. on CSVT, 6(3):243-250, June 1996.

[11] J. M. Shapiro. Embedded image coding using zerotrees of wavelet coefficients. IEEE Trans. on Sig. Proc., 41(12):3445-3462, Dec. 1993.

[12] P. G. Sherwood and K. Zeger. Progressive image coding for noisy channels. IEEE Sig. Proc. Letters, 4(7):189-191, July 1997.

[13] P. G. Sherwood and K. Zeger. Progressive image coding on noisy channels. In Proc. DCC '97, pages 72-81, 1997. 